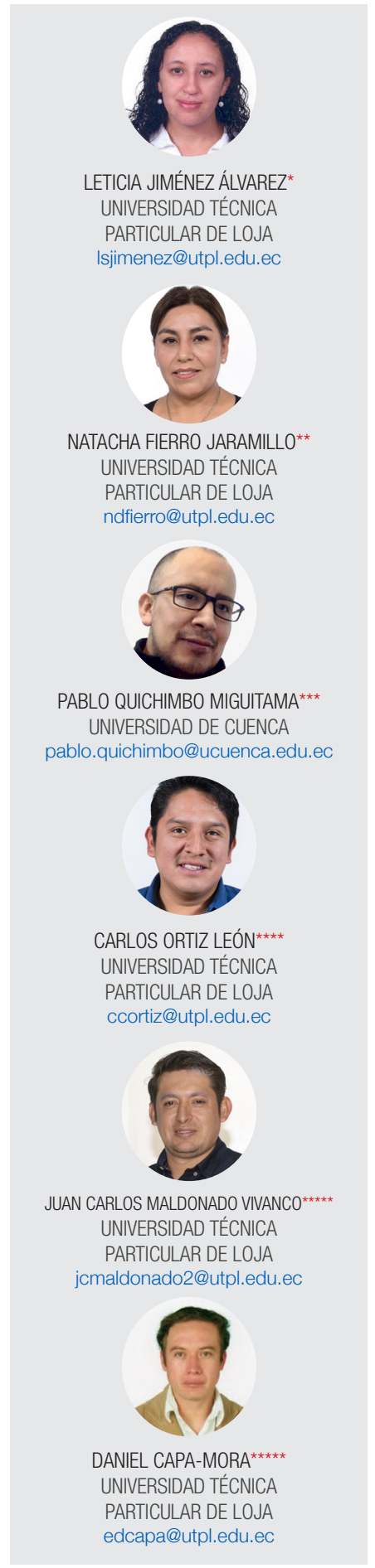

\title{
Incremento de la motivación a partir de la creación de recursos multimedia en un curso de la Ciencia del Suelo
}

\section{Increased motivation from the creation of multimedia resources in a course in Soil Science}

Recibido: 5 de junio de 2019 | Aprobado: 31 de octubre de 2019

\section{Resumen}

La enseñanza de la Ciencia del Suelo requiere herramientas dinámicas que permitan al estudiante participar de manera continua a lo largo del ciclo académico. El objetivo de esta investigación es integrar la tecnología a las estrategias de aprendizaje para aumentar la motivación y el rendimiento de los estudiantes. Se implementaron durante 16 semanas distintas estrategias en un curso de 34 estudiantes. Este estudio reporta el impacto de dos de ellas, que fueron la elaboración de un video y un póster. Los grupos que cumplieron con los requisitos asignados para la elaboración del video fueron motivados para trabajar con ayuda de técnicos en la producción y edición. Al final, se realizó una encuesta a los alumnos para conocer cuáles, según su percepción, fueron las mejores estrategias desarrolladas. Los datos fueron analizados descriptivamente. Tanto la elaboración del video como el póster complacieron a los estudiantes porque conllevaban un aprendizaje activo. Este estudio muestra que la motivación de los estudiantes puede aumentar en la medida en que se sienten involucrados en proyectos innovadores que utilizan recursos, herramientas y estrategias adecuadas. Esta implementación es útil no solo para el curso de la Ciencia del Suelo, sino, incluso, para otros campos, dependiendo de la naturaleza del grupo y de la asignatura.

Palabras clave: aprendizaje activo; estrategias de enseñanza; tecnología de la educación, educación superior; estilos de aprendizaje.

Doctora en Manejo y Gestión de Recursos Fitogenéticos, Universidad Técnica Particular de Loja. Para contactar a la autora: Isjimenez@utpl.edu.ec

** Mgtr. Gestión de la Formación: Planificación, Desarrollo y Evaluación de la Formación de Formadores, Universidad Técnica Particular de Loja. Para contactar a la autora: ndfierro@utpl.edu.ec

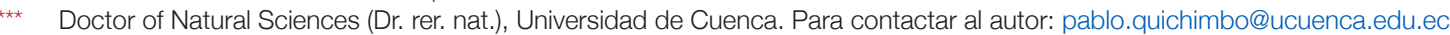

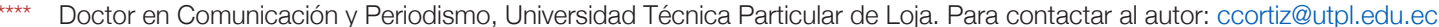

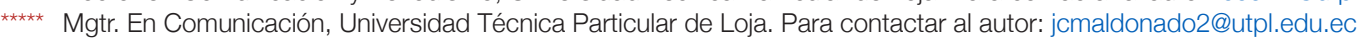

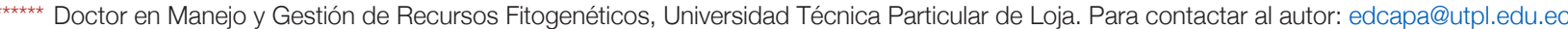




\section{Abstract}

The teaching of Soil Science requires dynamic tools that allow the student to participate continuously throughout the academic cycle. The objective of this research is to integrate technology into learning strategies with technology to improve student performance. The strategies were implemented for 16 weeks in a course of 34 students, and mainly two were applied, which were the preparation of a video and a poster; In the video, the groups that met the assigned requirements were motivated to work with the help of technicians in the production and editing of videos. In the end, a survey was carried out on the students to know which, according to their perception, were the best strategies developed. Data were analyzed descriptively. Both the making of the video and the poster pleased the students because it meant a greater option to learn. This study shows that student motivation can increase as they feel involved with innovative projects that use appropriate resources, tools and strategies. This implementation is useful not only for the course of Soil Science, but even for other fields, depending on the nature of the group and the subject.

Keywords: active learning; teaching strategies; educational technology, higher education; learning styles.

\section{Introducción}

A través de la enseñanza de la Ciencia del Suelo se contribuye a resolver problemas a escala global, regional, nacional y local (Hartemink, 2015; Mamo, et al., 2011), sin embargo, son poco alentadoras las noticias sobre la evolución de la enseñanza de la Ciencia del Suelo en las universidades (Macías, 2015). Esto indica que aún queda largo camino por recorrer en el ámbito de la enseñanza-aprendizaje de esta ciencia.

La enseñanza en la educación superior debe tener en cuenta las características específicas de la disciplina que se imparte (Berthiaume, 2009). Cualquiera que sea la forma utilizada, esta debe motivar a los estudiantes para que interactúen, participen y desarrollen de manera dinámica las competencias propias de cada materia. Dentro de los recursos que se pueden utilizar están los rompecabezas, mesas redondas, vídeos, trabajos de investigación, gráficos, animaciones, vídeo juegos (Annetta, 2008; Lom, 2012; Mohd, Asmadi, Azlina y Bhari, 2013), los cuales pueden constituirse en alternativas para mejorar el proceso de enseñanza - aprendizaje.

Varios de esos recursos permiten la interacción entre el estudiante, el docente, el medio real y los compañeros; y dan la oportunidad de que el alumno logre un aprendizaje activo y aumente su motivación, lo cual evita que el estudiante se limite a una participación pasiva. Varios artículos se refieren a la importancia de utilizar los vídeos en clase para el aprendizaje a través de la observación de temas específicos (Akbar, 2016), pero no es común encontrar la tarea de elaboración del vídeo en que el estudiante sea el actor principal que desarrolla todo el proceso (desde la investigación de los suelos del ecosistema para la elaboración del guion, hasta la selección del lugar para su grabación y posterior edición).

En el caso del póster, se suele utilizar como recurso didáctico para mejorar la enseñanza porque permite a los estudiantes exponer los resultados de un proyecto y permite que los demás compañeros también conozcan y comparen el producto final de su trabajo (Blanco y Prieto, 1989), además de fomentarse las competencias comunicativas al exponer una cantidad de información sintetizada en el producto elaborado. Muchas de estas actividades van de la mano con la tecnología, lo cual permite al estudiante explorar y descubrir su propio conocimiento de una forma más dinámica. Esto nos conduce a considerar, de acuerdo con Akbar (2016), que las prácticas pedagógicas deberían ir a la par con el impacto de las tecnologías. Este investigador piensa que la tecnología es una de las mejores herramientas educativas, porque estimula el uso de más de un sentido simultáneamente al incluir animaciones, fotografías, vídeo, audio (Aloraini, 2012; Eady y Lockyer, 2013). En épocas pasadas había menos acceso a los dispositivos tecnológicos (computadora y conexión a Internet), a diferencia de la actualidad, que los dispositivos móviles son más comunes y sus costos más bajos (Figueras-Maz, Ferrés y Mateus, 2018; Sunkel, Trucco y Espejo, 2013). Sin embargo, la tecnología por sí sola no conduce necesariamente a resultados 
de enseñanza efectivos, pero si contribuye a que la información esté más accesible, lo cual puede facilitar que los estudiantes estén más informados y disponibles para el aprendizaje (Annetta, 2008; Woolfitt, 2015).

Sumado a la tecnología está la motivación del estudiante, que se puede definir como el deseo de una persona por cumplir una meta, o como el motor para la acción que predispone a alguien a alcanzar un objetivo. La principal dificultad que encuentra el profesor en su quehacer diario es motivar al alumno. Entre los factores que influyen decisivamente a la motivación están el docente, los procedimientos de enseñanza que utiliza y el ambiente del aula (Biggs, 2006; López, 2007; Elizondo, Rodríguez y Rodríguez, 2018). Existen dos tipos de motivación, la motivación intrínseca, aquella en la que no hacen falta signos externos para que los estudiantes sientan un impulso genuino para aprender, la cual conduce al aprendizaje profundo y a un trabajo académico óptimo. Esta se da cuando el estudiante realiza una actividad sin esperar ningún incentivo externo. En cambio, la motivación extrínseca le da mayor interés y tiempo para cumplir aquellas actividades que serán evaluadas para satisfacer sus necesidades de estima. Hay dos categorías: el refuerzo positivo, en el que se da una recompensa material y, el refuerzo negativo, en la que se busca evitar un castigo (Biggs, 2006).

El incremento de la motivación se relaciona significativamente con continuar una carrera o terminar de manera exitosa una actividad; el desafío es estimular la motivación intrínseca para un aprendizaje independiente (Savage, Birch y Nousi, 2011; French, Immekus y Oakes, 2013). Por ello, debemos implementar estrategias de aprendizaje en el aula que mejoren la motivación de los alumnos y el posible aumento en el rendimiento académico, aunque no es una tarea sencilla y dista de ser simple, debido a que no existen fórmulas inmediatas y que se puedan acoplar a todos los estudiantes (Hoskins y Newstead, 2009). Esto conlleva a que es necesario innovar de acuerdo a las características específicas de cada caso. Además, los estudiantes necesitan tener disposición para trabajar en grupo y apoyarse en las habilidades individuales.

El propósito de esta investigación es motivar al uso de unas estrategias y de unos recursos en que los estudiantes sean protagonistas de su aprendizaje a través de herramientas tecnológicas, de la participación en escenarios reales y de la evaluación con un equipo multidisciplinario para que el estudiante desarrolle competencias profesionales al trabajar y ser evaluado de una manera más justa e imparcial (Hoskins y Newstead, 2009; Rodríguez, López y Mortera, 2017). La implementación llevada a cabo parte de que el referente es el estudiante con el fin de mejorar su rendimiento y el interés en las Ciencias del Suelo. Además, con este estudio se pretende conocer la percepción de los estudiantes sobre sus preferencias frente a las estrategias implementadas en el curso de Edafología, estrategias que pueden aplicarse en futuros cursos relacionados, incluso pueden aplicarse en otros campos dependiendo de la naturaleza de cada componente. A continuación, veremos la metodología que hemos aplicado en el estudio. Luego, dentro de ese acápite presentaremos las pautas y requisitos para la elaboración del video y de los pósteres. Por último, expondremos los resultados y las conclusiones a las que hemos llegado.

\section{Metodología}

Esta es una investigación descriptiva debido a que se muestra e interpreta el impacto de las actividades y recursos implementados. Además, se estudió la relación entre variables cuantificadas (Sarduy, 2006). La selección de la muestra fue de manera intencional (no probabilística). A la muestra seleccionada se le aplicó una encuesta a través de un cuestionario y una entrevista semiestructurada que permitieron conocer la percepción de los estudiantes sobre las estrategias aplicadas.

El curso de las Ciencias del Suelo se dictó en el ciclo de octubre 2017 - febrero 2018 con 34 estudiantes de la Carrera de Biología de la Universidad Técnica Particular de Loja. El curso tiene una duración de 16 semanas, con un total de 128 horas, de las cuales 64 fueron presenciales y las restantes constituían trabajo autónomo independiente; en los dos casos se utilizaron varias estrategias como trabajo de campo, laboratorio, trabajos grupales formales e informales, debates, participaciones continuas, entre otras (Ferreiro y Espino 2014). 
Esta investigación se enfoca principalmente en dos actividades que se trabajaron durante todo el ciclo, las cuales desarrollan distintas competencias de acuerdo a sus características, con el fin de impactar el aprendizaje de los estudiantes de acuerdo sus diferentes estilos y características (Quichimbo, Chérrez, Vanegas y Jiménez, 2018).

\section{El portafolio como herramienta tecnológica}

Entre las herramientas utilizadas está el portafolio digital, que constituye una herramienta tecnológica con el objeto de almacenar artículos, fotografías, presentaciones, trabajos, información en general, que facilita revisar los avances, evaluar las actividades o proyectos de clase y observar el desarrollo de las competencias.

\section{Elaboración de un vídeo}

Con la elaboración del vídeo se busca que el alumno adquiera varias competencias cognitivas, como el gestionar información derivada de fuentes diversas; disponer de habilidades informáticas; la generación de nuevas ideas; la toma de decisiones y organizar, planificar y ejecutar un proyecto (Sanz, 2010). A través de esta actividad también se pretende potenciar los estilos de aprendizaje de los estudiantes de las Ciencias del Suelo (Quichimbo et al., 2018; Jiménez, Vega, Capa-Mora, Fierro y Quichimbo, 2019), principalmente para quienes son activos y pragmáticos que se orientan mejor "haciendo". Se seleccionó esta opción para que, a través de la combinación de elementos como contenido, imagen y audio, se pueda apoyar al procesamiento de la información (Eady y Lockyer, 2013).

En la primera semana de clases se organizaron varios grupos de trabajo con 5 a 6 personas cada uno (Griffiths, 2009), a los que se asignó un tema específico, por ejemplo, suelos de "bosques secos", "páramos", "bosque tropical lluvioso". Como parte de la motivación se les explicó que quienes cumplieran semanalmente con las actividades asignadas (como la recopilación y lectura de artículos científicos y exposición de los avances en las fechas indicadas) trabajarían con un grupo de especialistas en la elaboración de sus vídeos, con el objetivo de que se comprometieran a realizar su mayor esfuerzo.

Con base en los avances logrados hasta la semana 8, se seleccionaron los grupos que cumplieron con lo asignado para que trabajasen con personas especializadas en la elaboración de vídeos. A diferencia de lo esperado, los grupos no seleccionados tomaron la elaboración del vídeo como un desafío y se esforzaron para entregar un buen producto. Cada grupo abrió un portafolio digital, en donde adicionaron fotografías, trabajos de clase, presentaciones, apuntes y artículos científicos con las ideas principales subrayadas que se utilizarían en el vídeo sobre el tema asignado. Los estudiantes trabajaron en la elaboración del guion, en la selección del lugar, en la grabación y posterior edición (Figura 1).

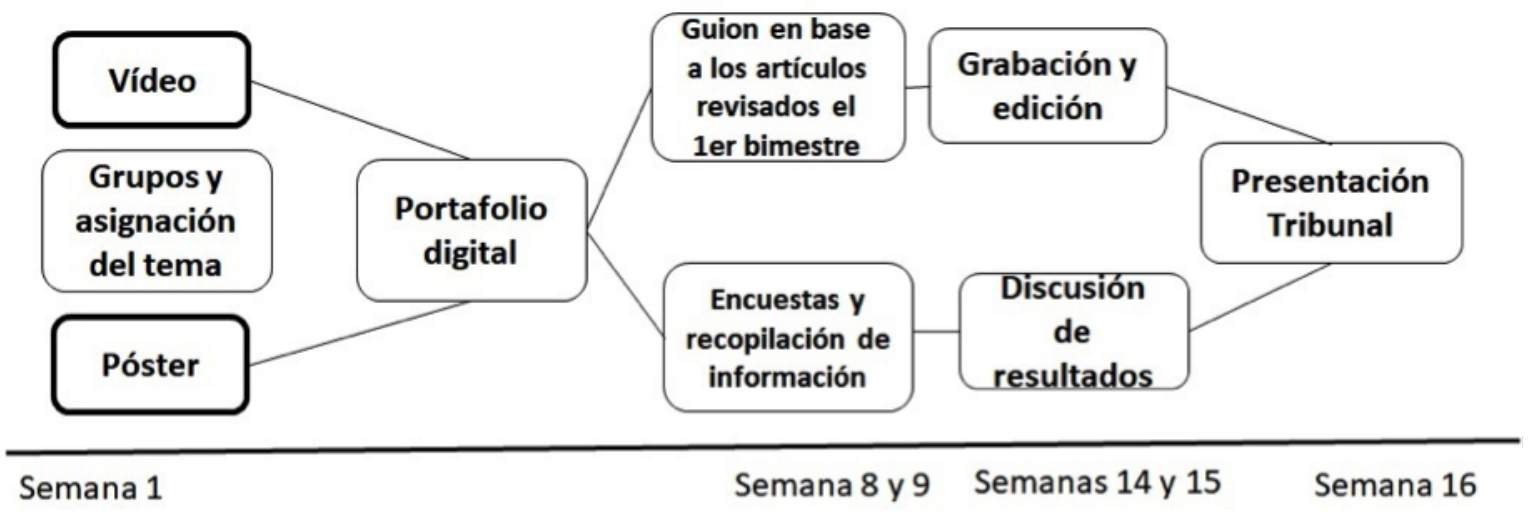

Figura 1. Esquema de las actividades realizadas en el curso de Ciencias del Suelo 
La evaluación del vídeo la realizó un grupo multidisciplinario de cuatro docentes, utilizando una rúbrica (Anexo 1) con cinco parámetros como: 1. la organización y desarrollo del contenido; 2. la información científica utilizada en el desarrollo del vídeo; 3. la presentación oral y duración del tema; 4. Creatividad y 5. el audio y calidad de la imagen. La valoración de esta estrategia fue de 6 puntos en cada bimestre o 12 puntos en el ciclo (un ciclo está conformado por dos bimestres) del total de 40 puntos. La participación de otros docentes externos al tema se incluyó para agregar nuevas dimensiones a la actividad de aprendizaje (Davis y Wilcock, 2003).

\section{Póster}

A través del póster se busca que el alumno adquiera competencias cognitivas como la búsqueda y gestión de información derivada de fuentes diversas; la generación de nuevas ideas; adquirir conocimientos de diversos ámbitos de estudio y organizar y sintetizar la información (Sanz, 2010). Además, potencia los diversos estilos de aprendizaje de los estudiantes de las Ciencias del Suelo, en especial los reflexivos y teóricos que se orientan mejor "pensando", como conlleva en la elaboración del póster.

Con esta estrategia los estudiantes tienen la oportunidad de experimentar con el entorno e investigar sobre el manejo del suelo, mejorando la experiencia de aprendizaje del alumno. Los mismos grupos que trabajaron la estrategia anterior, realizaron varias encuestas referentes a la temática "conocimiento local de la gente sobre el manejo del suelo". La encuesta estuvo conformada por 35 preguntas que englobaron los indicadores de la fertilidad de los suelos. Una vez obtenidos los resultados, los tabularon y contrastaron a partir del conocimiento científico. La información sistematizada de esta investigación la plasmaron en un póster, el cual fue presentado en una exposición con el mismo tribunal utilizado para la evaluación del vídeo.

Cada grupo fue evaluado aproximadamente por 20 minutos a través de la presentación de los principales resultados y respondieron preguntas. La evaluación del póster se realizó con base en una rúbrica (Anexo 2), incluyó: 1. Organización y desarrollo del contenido, 2. Dominio del tema y 3. Respuestas a las preguntas; con una valoración de 6 puntos. Cada docente llenó la rúbrica de cada grupo y de cada estudiante y al final emitieron un comentario general de las estrategias utilizadas (Figura 1).

En el caso de las dos estrategias implementadas, se revisó el avance cada 15 días con exposiciones cortas para orientary retroalimentar a los estudiantes, además para mantener su motivación y promover el aprendizaje.

Al finalizar el curso (16 semanas), se realizó una encuesta opcional a los estudiantes para conocer su percepción frente a las estrategias utilizadas. En ella se consultó, entre otras preguntas: ¿Qué estrategia consideras que te motivó más a aprender?; ¿La idea de ser escogido para obtener ayuda especializada te motivó a esforzarte más? En caso que no hayas tenido ayuda especializada para la elaboración del vídeo, eso: ¿te desanimó?, ¿te fue indiferente? ¿fue un desafío? Otras preguntas incluidas fueron: ¿Qué aspectos resaltas de la elaboración del vídeo?; ¿Qué otras estrategias sugieres que se deban aplicar en la clase? (Anexo 3). Además, se entrevistó a algunos estudiantes para conocer más a detalle su opinión. También se consideró el criterio de los docentes que participaron en la evaluación.

\section{Análisis de datos}

Los resultados obtenidos se analizaron de manera descriptiva, comparando el porcentaje de las estrategias utilizadas de un curso de la Ciencia del Suelo en base a la percepción y rendimiento que obtuvieron los estudiantes.

\section{Resultados}

Los resultados nos indican que, de las estrategias utilizadas en el componente de Edafología, los estudiantes valoraron mejor el vídeo y el póster con el 61 \% (Figura 2). Si bien, el trabajo se duplica para el profesor y el alumno, la realización de las dos actividades también significa mayor opción para el aprendizaje de estudiantes con diversos estilos de aprendizaje (Quichimbo, Chérrez y Jiménez, 2016; Jiménez et al., 2019).

Al comparar las calificaciones entre el primer y segundo bimestre, se observó que el 74 \% de los 
estudiantes mejoraron sus notas principalmente entre 1 y 4 puntos, debido a que el vídeo y póster los presentaron al finalizar el ciclo y esa nota correspondió al segundo bimestre. También, se observó que el promedio general final (sobre 40 puntos) de este curso, comparado con el del año pasado con el mismo número de estudiantes, pero con estrategias diferentes, fue mayor, 30,20 frente a 25,94 puntos, respectivamente. Estos promedios son evidencias de que el objetivo de la implementación de estrategias innovadoras y de participación activa promovió la mejora del aprendizaje de los estudiantes en las Ciencias del Suelo, lo cual concuerda con Kern y Carpenter (1984), quienes indican que el brindar un ambiente de trabajo en el aula y fuera de ella como factor para la motivación de los estudiantes, ayudará en su mejora académica. El implementar estrategias y actividades que conllevan el uso de la investigación y la tecnología con un propósito extrínseco, la participación de trabajar y ser evaluados por expertos aumenta la motivación de los estudiantes y ayuda a su mejora académica.

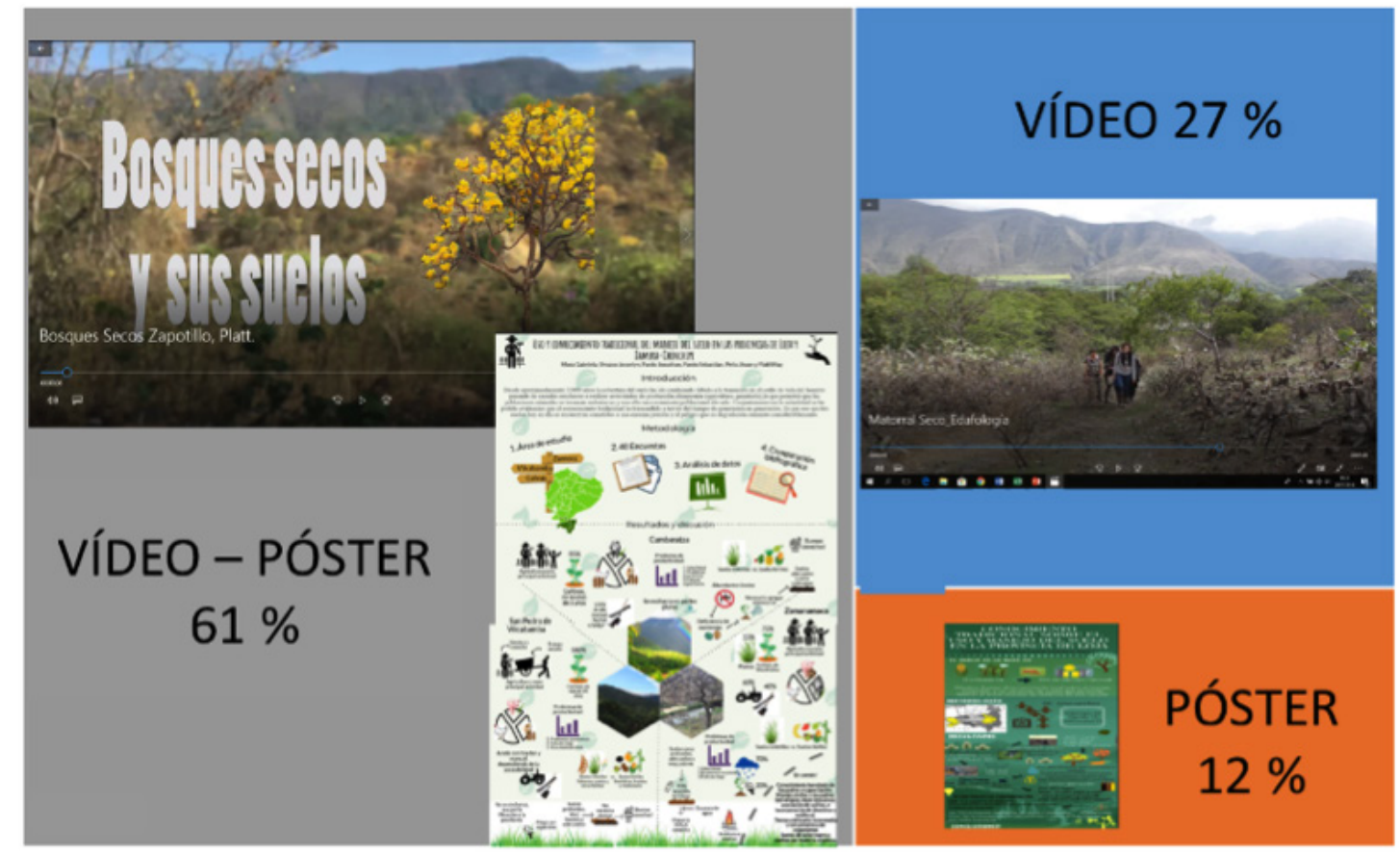

Figura 2. Porcentajes de preferencia de las estrategias utilizadas en un curso de las Ciencias del Suelo

Un grupo menor con el $27 \%$ prefirió como estrategia solo el vídeo, esto puede ser porque ellos debían realizar la producción con ayuda de docentes y estudiantes externos, resultando una estrategia atractiva debido a que se realiza en un entorno informal (Guo, Kim y Rubin, 2014). Del grupo que recibió ayuda en su trabajo, el $88 \%$ de los estudiantes mencionó que la elaboración del vídeo con ayuda especializada los motivó a esforzarse más, lo que demuestra que la presencia de expertos externos como evaluadores aumenta la motivación a lograr metas más altas. Un hallazgo de esta investigación es que, el grupo que no recibió ayuda especializada para realizar el vídeo, también mencionó con el porcentaje más alto, que el trabajar sin apoyo fue un reto para presentar un mejor trabajo; menor al 15 \% se desanimó por no haber sido seleccionado y el restante $6 \%$ le fue indiferente, aunque el promedio de nota final fue menor a los que si obtuvieron ayuda. Este sencillo caso demuestra que la motivación se logra a través de estímulos positivos o negativos, situación última que incluye la resiliencia, capacidad humana para salir de abajo y vencer los obstáculos (Stanford y Connie, 2009). 


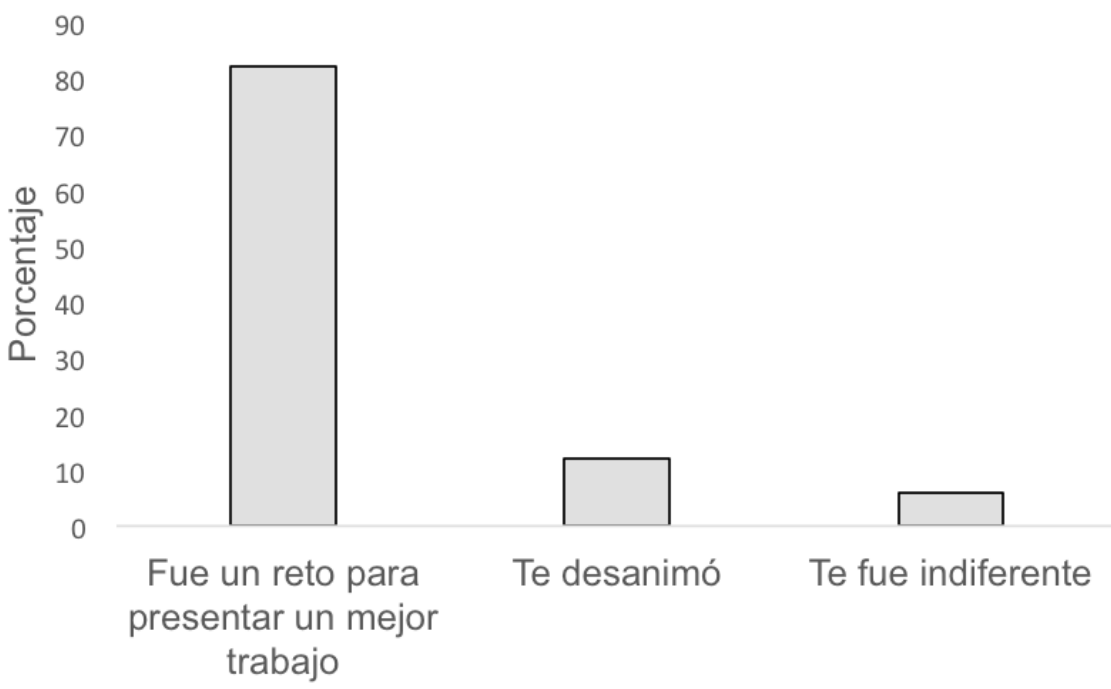

Figura 3. Respuesta en porcentajes de estudiantes que contestaron la pregunta. En caso que no hayas tenido ayuda especializada para la elaboración del vídeo, ¿cómo te sentiste?

El vídeo fue una tarea que ofreció oportunidades, pero al mismo tiempo resultó un desafío, no solo para alcanzar una mejor nota, sino también para ser los mejores; a pesar de que el principal objetivo de los estudiantes era asegurar altas calificaciones, demostraron entusiasmo e interés en la realización del vídeo.

Tabla 1. Ventajas y desventajas del vídeo y póster al aplicarlas en clases

\begin{tabular}{|c|c|c|}
\hline Actividad & Ventajas & Desventajas \\
\hline Vídeo & $\begin{array}{l}\text { - Tiene la opción de grabar varias veces hasta } \\
\text { lograr las mejores tomas } \\
\text { - Puede editarlo hasta obtener un producto de } \\
\text { - } \text { mejor calidad } \\
\text { - } \text { de manera lica la temática en un tiempo reducido } \\
\text { - } \text { Motiva a los estudiantes a realizar las } \\
\text { - } \quad \text { Petividades } \\
\text { - Permite trabajar con diversos elementos }\end{array}$ & $\begin{array}{l}\text { - Requiere competencias desarrolladas en la } \\
\text { - elaboración de productos multimedia } \\
\text { - } \quad \text { temor conveniente para personas con } \\
\text { - Mayor trabajo y tiempo en la producción } \\
\text { del vídeo }\end{array}$ \\
\hline $\begin{array}{l}\text { Póster } \\
\text { (trabajo de } \\
\text { campo y } \\
\text { escritorio) }\end{array}$ & $\begin{array}{l}\text { - Permite la construcción de relaciones } \\
\text { personales con una comunidad local y } \\
\text { aprender directamente desde múltiples } \\
\text { perspectivas } \\
\text { - } \quad \text { Contrastar la información local con la científica } \\
\text { - } \text { Recurso económico y accesible } \\
\text { - } \quad \text { Sintetiza el contenido } \\
\text { - } \\
\text { Permite explicar los contenidos mientras el } \\
\text { observador lo lee }\end{array}$ & $\begin{array}{l}\text { - Límite de imágenes y texto } \\
\text { - Menor atención del observador frente al } \\
\text { vídeo }\end{array}$ \\
\hline
\end{tabular}


El 12 \% opinó que la elaboración del póster fue la mejor actividad en comparación al vídeo. Sin embargo, el póster presentó otras fortalezas frente a la otra actividad realizada (tabla 1). En este caso, la comunidad se convirtió para el grupo en un escenario real, lo cual le permite conocer la problemática del manejo de los suelos y con ello discutir posibles soluciones como un desafío local, que le permitirá al estudiante ganar experiencia en un contexto holístico (Field, et al., 2011; Field, Yates, Koppi, McBratney y Jarret, 2017).

\section{Conclusiones}

Una observación recurrente por los estudiantes en el caso de las dos estrategias fue la poca colaboración de algunos miembros de los grupos, por lo que los estudiantes sugieren conformarlos por afinidad y de menor número, la organización y funcionamiento de los grupos pequeños es una variable clave en el éxito y desempeño individual. No basta con sugerirles trabajar en grupo, si no poseen habilidades previas para hacerlo. La formación previa para el trabajo colaborativo los hará buenos trabajadores individuales, y a la vez, las formas de evaluación comunes individualistas se irán sustituyendo por una evaluación de logro grupal.

De acuerdo con la literatura contrastada sobre el trabajo en equipos cooperativos, el mejor número de integrantes para grupos de trabajo se sitúa en tres, máximo cuatro. En segundo lugar, es necesario que entre ellos se dé una diversificación de roles y puedan ser evaluados de acuerdo al rol que desempeñó cada uno. Esto permite una mejor organización por el hecho de que nadie hará la misma tarea que otro, y de la misma manera, esto facilitará la interacción positiva entre ellos. Estas son las claves para el buen funcionamiento de los pequeños grupos. También el hecho de haber elaborado un portafolio en las actividades (vídeo y póster), que fue revisado cada 15 días con exposiciones cortas de los avances, permitió realizar retroalimentación para fortalecer las actividades realizadas y mejorar aquellas que no estaban claras en el grupo, aspirando a lograr un aprendizaje más profundo de las Ciencias del Suelo.
Con este trabajo se ha proporcionado una breve visión de dos estrategias que se pueden utilizar en el aula de clase para mejorar la motivación del estudiante. El utilizar estrategias que incluyan tecnología, texto, imágenes, vídeo, (multimedia) es una oportunidad para hacer que la metodología sea más variada y activa, lo cual permite que el aprendizaje tenga un mayor impacto, que los estudiantes desarrollen un rol más protagónico, que se sientan más motivados y que desarrollen competencias creativas en el proceso de enseñanza - aprendizaje. Pero es muy importante que las estrategias que se utilicen estén conectadas a los objetivos de aprendizaje del curso y que fomenten un desempeño independiente y colaborativo a la vez, en la cual el estudiante no sea un destinatario pasivo de la información, sino protagonista de su proceso educativo. Es recomendable utilizar actividades variadas que promuevan la atención y motivación de los estudiantes, la cual se incrementa cuando se sienten involucrados y evaluados de una manera justa y por expertos.

\section{Referencias bibliográficas}

Akbar, M. (2016). Digital technology shaping teaching practices in higher education. Frontiers, 3, 1-5. doi.org/10.3389/fict.2016.00001

Aloraini, S. (2012). The impact of using multimedia on students' academic achievement in the College of Education at King Saud University. Journal of King Saud University - Languages and Translation, 24, 75-82. doi.org/10.1016/j. jksult.2012.05.002

Annetta, L. (2008). Video Games in Education: Why they should be used and how they are being used. Theory Into Practice, 47, 229239. Disponible en http://www.jstor.org/ stable/40071547

Berthiaume, D. (2009). Teaching in the disciplines. En H. Fry, S. Ketteride, S. Marshall (Eds.), A Handbook for teaching and learning in higher education, enhancing academic practice (pp. 215 - 225). New York and London: Routledge.

Biggs, J. (2006). Calidad del aprendizaje universitario. Madrid: Narcea. 
Blanco, A. y Prieto, T. (1989). El póster como recurso didáctico desde una perspectiva de la enseñanza-aprendizaje. Investigación en la Escuela, 9, 85-86.

Davis, C. y Wilcock, E. (2003). Teaching materials using case studies. UK: UK Centre for Materials Education, 1-20. Disponible en http://www. materials.ac.uk/guides/casestudies.asp

Eady, M. y Lockyer, L. (2013). Tools for learning: technology and teaching strategies. Learning to Teach in the Primary School. Australia: Queensland University of Technology.

Elizondo, A., Rodríguez, J. y Rodríguez, I. (2018). La importancia de la emoción en el aprendizaje: Propuestas para mejorar la motivación de los estudiantes. Cuaderno de Pedagogía Universitaria 15 (29) 1-11.

Field, D., Koppi, A., Jarrett, L., Abbott, L., Cattle, S., Grant, C., Mcbratney, A., ... y Weatherley, A. (2011). Soil Science teaching principles. Geoderma, 167-168, 9-14. doi.org/10.1016/j. geoderma.2011.09.017

Field, D., Yates, D., Koppi, A., McBratney, A. y Jarret, L. (2017). Framing a modern context of soil science learning and teaching. Geoderma, 289, 117 -123. doi.org/10.1016/j. geoderma.2016.11.034

Figueras-Maz, M., Ferrés, J. y Mateus, J. C. (2018). Percepción de los/as coordinadores/as de la innovación docente en las universidades españolas sobre el uso de dispositivos móviles en el aula. Prisma Social, 160-179.

French, B., Immekus, J. y Oakes, W. (2013). An Examination of Indicators of Engineering Students' Success and Persistence. Journal of Engineering Education, 94(4).

Griffiths, S. (2009). Teaching and learning in small groups. En H. Fry, S. Ketteride, S. Marshall (Eds.), A Handbook for teaching and learning in higher education, enhancing academic practice (pp. 72 - 84). New York and London: Routledge.
Guo, P., Kim, J. y Rubin, R. (2014). How video production affects student engagement: an empirical study of MOOC videos. Proceedings of the first ACM conference on Learning scale conference, March 04 - 05, 2014, Atlanta, GA, pp 41-50. ACM. doi. org/10.1145/2556325.2566239

Hartemink, A. (2015). On global soil science and regional solutions. Geoderma Regional, 5, 1-3. doi.org/10.1016/j.geodrs.2015.02.001

Hoskins, S. y Newstead, S. (2009). Encouraging student motivation. En H. Fry, S. Ketteride, S. Marshall (Eds.), A Handbook for teaching and learning in higher education, enhancing academic practice (pp. 27 - 29). New York and London: Routledge.

Jiménez, L., Vega, N., Capa-Mora, D., Fierro, N. y Quichimbo, P. (2019). Estilos y estrategias de enseñanza-aprendizaje de estudiantes universitarios de la Ciencia del Suelo. Revista Electrónica de Investigación Educativa, 21, e04. doi.org/10.24320/redie.2019.21. e04.1935

Kern, E. y Carpenter, J. (1984). Enhancement of student values, interests and attitudes in earth science through a field-oriented approach. Journal of Geological Education, 32, 209 305.

Lom, B. (2012). Classroom activities: simple strategies to incorporate student-centered activities within undergraduate science lectures. The Journal of Undergraduate Neuroscience Education, 11(1), A64-A71.

López, F. (2007). Metodología participativa en la enseñanza universitaria ( $2^{a}$ ed.). Madrid: Narcea.

Macías, F. (2015). Retos y oportunidades de la ciencia del suelo: aprendiendo de los suelos, aprendiendo de la naturaleza. Spanish Journal of Soil Science, 5, 1- 11. Disponible en file://C:/Users/Isjimenez/Downloads/11972914-1-PB.pdf 
Mamo, M., Ippolito, J., Kettler, T., Reuter, R., McCallister, D., Morner, P., ... y Blankenship, E. (2011). Learning gains and response to digital lessons on soil genesis and development Journal of Geoscience Education, 59(4), 194204. DOI: $10.5408 / 1.3651402$

Mohd, M. Z., Asmadi, A., Azlina, N. y Bhari, A. (2013). Relationship Between the Multimedia Technology and Education in Improving Learning Quality. Procedia Social and Behavioral Science, 90, 351 - 355. doi. org/10.1016/j.sbspro.2013.07.102

Quichimbo, P., Chérrez, M. y Jiménez L. (2016). Students' learning styles in soil science: Ecuadorian case study. Ecuador es Calidad, 45-50. Disponible en http://www.agrocalidad. gob.ec/revistaecuadorescalidad/index.php/ revista/article/viewFile/47/88

Quichimbo, P., Chérrez M. E., Vanegas, R. y Jiménez, L. (2018). Relationship between Ecuadorian student's learning styles and academic perfomance in soil science. Ciencia del Suelo, 35(1) 196-203.

Rodríguez, R., López, B. y Mortera, F. (2017). El video como recurso educativo abierto y la enseñanza de matemáticas. Revista Electrónica de Investigación Educativa, 19(3), 92-100. doi. org/10.24320/redie.2017.19.3.936
Savage, N., Birch, R. y Noussi, E. 2011. Motivation of engineering students in higher education. Engineering Education, 6(2) 39-46.

Sanz, M. L. (2010). Competencias cognitivas en Educación Superior. Madrid: Narcea.

Sarduy, Y. D. (2006). El análisis de información $y$ las investigaciones cuantitativa $y$ cualitativa. Escuela Nacional de Salud Pública. Disponible en https://www. scielosp.org/scielo.php?pid=S0864$34662007000300020 \&$ script $=$ sci $_{\text {_ }}$ arttext\&tlng=pt

Stanford T. G y Connie, M. (2009). Psychology of Success: Overcoming Barriers to Pursuing Further Education. The Journal of Continuing Higher Education, 57 (1) 10-21.

Sunkel, G., Trucco, D. y Espejo, A. (2013). La integración de las tecnologías digitales en las escuelas de América Latina y el Caribe, una mirada multidimensional. Chile: CEPAL

Woolfitt, Z. (2015). The effective use of video in higher education. Lectoraat Teaching. Learning and Technology Inholland University of Applied Sciences, 1-49. Disponible en https://www.inholland.nl/media/10230/theeffective-use-of-video-in-higher-educationwoolfitt-october-2015.pdf

\section{Anexo 1}

Rúbrica para la evaluación de vídeo (Edafología)

\section{Criterios:}

1. Organización y desarrollo del contenido

2. Fuentes bibliográficas utilizadas (Bibliografía actual y científica)

3. Presentación oral y duración

\section{Creatividad}

5. Audio y calidad de la imagen 


\begin{tabular}{|c|c|c|c|}
\hline & Avanzado (2.4 puntos) & Medio (1.6 puntos) & Básico (0.8 puntos) \\
\hline \multirow[t]{2}{*}{$\begin{array}{l}\text { Organización, } \\
\text { desarrollo del } \\
\text { contenido }\end{array}$} & $\begin{array}{l}\text { El tema está claro y bien } \\
\text { enfocado. Destaca la idea } \\
\text { principal y es respaldada con } \\
\text { información detallada. } \\
\text { Describe las principales } \\
\text { características físico- químicas } \\
\text { edáficas del ecosistema } \\
\text { asignado (al menos } 5 \text { de } \\
\text { ellas). Explica claramente la } \\
\text { relación suelo - planta en el } \\
\text { ecosistema. }\end{array}$ & $\begin{array}{l}\text { La idea principal es } \\
\text { medianamente clara, se } \\
\text { necesita mayor información de } \\
\text { apoyo. } \\
\text { Describe las principales } \\
\text { características físico- químicas } \\
\text { edáficas del ecosistema } \\
\text { asignado (de } 3 \text { a } 5 \text { de ellas). } \\
\text { Explica de manera más o } \\
\text { menos clara la relación suelo - } \\
\text { planta en el ecosistema. }\end{array}$ & $\begin{array}{l}\text { La idea principal no es clara. Parece } \\
\text { haber poca información recopilada y } \\
\text { está desordenada. } \\
\text { Menciona una o ninguna causa, } \\
\text { efecto y solución al problema. } \\
\text { Describe una o dos de las } \\
\text { características físico- químicas } \\
\text { edáficas del ecosistema. Explica } \\
\text { de manera muy general y confusa } \\
\text { la relación suelo - planta en el } \\
\text { ecosistema. }\end{array}$ \\
\hline & Avanzado (2.4 puntos) & Medio (1.6 puntos) & Básico (0.8 puntos) \\
\hline \multirow[t]{2}{*}{$\begin{array}{l}\text { Fuentes } \\
\text { bibliográficas } \\
\text { utilizadas } \\
\text { (Bibliografía actual } \\
\text { y científica) }\end{array}$} & $\begin{array}{l}\text { Utiliza fuentes confiables como } \\
\text { artículos científicos, libros o } \\
\text { información actualizada de } \\
\text { instituciones y organismos } \\
\text { especializados o información de } \\
\text { su investigación (al menos } 15 \\
\text { referencias bibliográficas). }\end{array}$ & $\begin{array}{l}\text { Utiliza fuentes confiables como } \\
\text { artículos científicos, libros o } \\
\text { información actualizada de } \\
\text { instituciones y organismos } \\
\text { especializados o información de } \\
\text { su investigación (entre } 8 \text { y } 14 \\
\text { referencias bibliográficas). }\end{array}$ & $\begin{array}{l}\text { Utiliza fuentes confiables como } \\
\text { artículos científicos, libros o } \\
\text { información actualizada de } \\
\text { instituciones y organismos } \\
\text { especializados o información de su } \\
\text { investigación (Menos de } 8 \text { referencias } \\
\text { bibliográficas). }\end{array}$ \\
\hline & Avanzado (2.4 puntos) & Medio (1.6 puntos) & Básico (0.8 puntos) \\
\hline \multirow[t]{2}{*}{$\begin{array}{l}\text { Presentación oral } \\
\text { y duración }\end{array}$} & $\begin{array}{l}\text { Muy buena presentación, } \\
\text { mantiene en todo momento la } \\
\text { atención de la audiencia. La } \\
\text { duración está de acuerdo a lo } \\
\text { establecido. }\end{array}$ & $\begin{array}{l}\text { Existen algunos problemas } \\
\text { en la presentación, pero no } \\
\text { se pierde el interés de la } \\
\text { audiencia. Presenta en menor } \\
\text { tiempo o excede lo establecido. }\end{array}$ & $\begin{array}{l}\text { Existen varios problemas en la } \\
\text { presentación y no mantiene el interés } \\
\text { de la audiencia. Presenta el vídeo } \\
\text { en la mitad del tiempo establecido o } \\
\text { excede el doble de tiempo. }\end{array}$ \\
\hline & Avanzado (2.4 puntos) & Medio (1.6 puntos) & Básico (0.8 puntos) \\
\hline \multirow[t]{2}{*}{ Creatividad } & $\begin{array}{l}\text { El vídeo tiene un alto grado de } \\
\text { originalidad. Utiliza muchos } \\
\text { recursos didácticos en la } \\
\text { presentación del vídeo. }\end{array}$ & $\begin{array}{l}\text { El vídeo es parcialmente } \\
\text { original. Utiliza varios recursos } \\
\text { en la presentación del vídeo. }\end{array}$ & $\begin{array}{l}\text { El vídeo es poco original. Utiliza } \\
\text { solo dos o tres recursos en la } \\
\text { presentación del vídeo. }\end{array}$ \\
\hline & Avanzado (2.4 puntos) & Medio (1.6 puntos) & Básico (0.8 puntos) \\
\hline $\begin{array}{l}\text { Audio y calidad de } \\
\text { la imagen }\end{array}$ & $\begin{array}{l}\text { El audio y la imagen son claras, } \\
\text { no existen interrupciones, el } \\
\text { volumen es adecuado. }\end{array}$ & $\begin{array}{l}\text { El audio y la imagen es clara } \\
\text { en ciertas partes del vídeo, } \\
\text { pero existen partes en que } \\
\text { disminuye la calidad; no existen } \\
\text { interrupciones, el volumen es } \\
\text { adecuado. }\end{array}$ & $\begin{array}{l}\text { El audio y la imagen son poco claras, } \\
\text { existen interrupciones, el volumen es } \\
\text { muy bajo. }\end{array}$ \\
\hline TOTAL & Hasta 12 puntos & Hasta 8 puntos & Hasta 4 puntos \\
\hline
\end{tabular}




\section{Anexo 2}

Rúbrica para la evaluación del póster (Edafología)

\section{Criterios:}

1. Organización y desarrollo del contenido, presentación del póster

\section{Dominio del tema}

3. Respuestas a las preguntas

\begin{tabular}{|c|c|c|c|}
\hline & Avanzado (2 puntos) & Medio (1.33 puntos) & Básico (0.66 punto) \\
\hline $\begin{array}{l}\text { Organización, } \\
\text { desarrollo del } \\
\text { contenido. } \\
\text { Presentación en } \\
\text { PowerPoint }\end{array}$ & $\begin{array}{l}\text { El tema está claro y bien } \\
\text { enfocado. Destaca la idea } \\
\text { principal y es respaldada con } \\
\text { información detallada. } \\
\text { La presentación es didáctica y } \\
\text { mantiene la atención del resto de } \\
\text { estudiantes. }\end{array}$ & $\begin{array}{l}\text { La idea principal es medianamente } \\
\text { clara, se necesita mayor } \\
\text { información de apoyo. } \\
\text { La presentación es en parte } \\
\text { didáctica y mantiene la atención } \\
\text { del resto de estudiantes. }\end{array}$ & $\begin{array}{l}\text { La idea principal no es clara. } \\
\text { Parece haber poca información } \\
\text { recopilada y está desordenada. } \\
\text { La presentación no es didáctica y } \\
\text { mantiene en parte la atención del } \\
\text { resto de estudiantes. }\end{array}$ \\
\hline & Avanzado (2 puntos) & Medio (1.33 puntos) & Básico (0.66 punto) \\
\hline $\begin{array}{l}\text { Dominio del } \\
\text { tema (explica, } \\
\text { discute, detalla, } \\
\text { ejemplifica, } \\
\text { argumenta) }\end{array}$ & $\begin{array}{l}\text { Presenta los resultados de } \\
\text { manera clara y coherente. } \\
\text { Demuestra dominio del tema, } \\
\text { presenta claridad y profundidad } \\
\text { en lo expresado, capacidad } \\
\text { de síntesis. Discute utilizando } \\
\text { referencias bibliográficas } \\
\text { científicas. }\end{array}$ & $\begin{array}{l}\text { Presenta los resultados de manera } \\
\text { más o menos clara y coherente. } \\
\text { Demuestra en parte dominio del } \\
\text { tema; presenta medianamente } \\
\text { profundidad en lo expresado, } \\
\text { capacidad de síntesis. Discute } \\
\text { utilizando pocas referencias } \\
\text { bibliográficas científicas. }\end{array}$ & $\begin{array}{l}\text { Presenta los resultados de } \\
\text { manera confusa y poco coherente. } \\
\text { Demuestra poco o nada de } \\
\text { dominio del tema. Discute sin } \\
\text { utilizar referencias bibliográficas } \\
\text { científicas. }\end{array}$ \\
\hline & Avanzado (2 puntos) & Medio (1.34 puntos) & Básico (0.67 punto) \\
\hline $\begin{array}{l}\text { Respuestas a las } \\
\text { preguntas }\end{array}$ & $\begin{array}{l}\text { Responde todas las preguntas de } \\
\text { manera adecuada, precisa, clara } \\
\text { y pertinente. }\end{array}$ & $\begin{array}{l}\text { Responde algunas preguntas } \\
\text { de manera adecuada, clara y } \\
\text { pertinente. }\end{array}$ & $\begin{array}{l}\text { Responde pocas preguntas } \\
\text { de manera adecuada, clara y } \\
\text { pertinente. }\end{array}$ \\
\hline TOTAL & Hasta 6 puntos & Hasta 4 puntos & Hasta 2 puntos \\
\hline
\end{tabular}




\section{Anexo 3}

\section{TITULACIÓN DE}

COMPONENTE DE EDAFOLOGÍA

Nombre:

1. ¿Qué estrategia consideras que te motivó más a aprender?:

Vídeo ( ) Póster ( ) Las dos anteriores ( ) Otras ( )

¿Por qué?

2. ¿La idea de que tu proyecto sea escogido para obtener seguimiento por especialistas te motivó a esforzarte más?:

Sí ( ) No ( )

¿Por qué?

3. En caso que no hayas tenido ayuda especializada para la elaboración del vídeo, eso:

\begin{tabular}{|l|c|l|c|}
\hline Te desanimó & $($ ) & Te fue indiferente & $(~)$ \\
\hline $\begin{array}{l}\text { Fue un reto para presentar un mejor } \\
\text { trabajo }\end{array}$ & $(\quad)$ & Otra opinión (¿Cuál?) & $(\quad)$ \\
\hline
\end{tabular}

4. ¿Qué aspectos resaltas de la elaboración del vídeo?

\begin{tabular}{|l|c|l|c|}
\hline Trabajo en equipo & $(\quad$ Competencia entre grupos & $($ ) \\
\hline Adquisición de nuevas competencias & $(\quad)$ & Reforzó los conocimientos & $(\quad$ ) \\
\hline Autoaprendizaje & $(\quad)$ & Aprendizaje integral \\
\hline Otras (¿Cuáles?)
\end{tabular}

5. ¿Con la elaboración del vídeo consideras que mejoró tu rendimiento?

Nada ( ) Poco ( ) Mucho ( ) Más de lo que esperaba ( )

6. ¿Qué otras estrategias sugieres que se deban aplicar en la clase?

7. ¿Qué estrategias consideran que se deberían eliminar?

¿Por qué? 